\title{
Lidil
}

Revue de linguistique et de didactique des langues

46 | 2012

Typologie et description linguistiques

\section{Une approche typologique fonctionnelle de la voix passive en français et en espagnol}

\section{Roser Gauchola}

\section{OpenEdition}

\section{Journals}

Édition électronique

URL : http://journals.openedition.org/lidil/3250

DOI : $10.4000 /$ lidil.3250

ISSN : 1960-6052

\section{Éditeur}

UGA Éditions/Université Grenoble Alpes

\section{Édition imprimée}

Date de publication : 15 décembre 2012

Pagination : 153-173

ISBN : 978-2-84310-236-3

ISSN : 1146-6480

\section{Référence électronique}

Roser Gauchola, « Une approche typologique fonctionnelle de la voix passive en français et en espagnol », Lidil [En ligne], 46 | 2012, mis en ligne le 15 juin 2014, consulté le 30 avril 2019. URL : http:// journals.openedition.org/lidil/3250 ; DOI : 10.4000/lidil.3250 


\title{
Une approche typologique fonctionnelle de la voix passive en français et en espagnol ${ }^{1}$
}

\author{
Roser Gauchola*
}

\begin{abstract}
RÉSUMÉ
En typologie linguistique fonctionnelle, la voix passive est conçue comme une des structures dont disposent certaines langues pour résoudre un problème cognitif et communicatif universel, la fonction de «participation», i.e. la relation entre un verbe et ses satellites nominaux. Dans cet article, nous présentons une caractérisation fonctionnelle de la voix passive et illustrons la démarche en analysant la contribution de la voix passive à l'expression de la fonction de participation dans deux langues typologiquement proches et génétiquement apparentées, le français et l'espagnol.
\end{abstract}

\begin{abstract}
In functional linguistic typology, passive voice is conceived as one of the structures available in certain languages to solve a universal cognitive and communicative problem, the function of "participation", i.e. the relation between a verb and its nominal satellites. In this paper, we present a functional characterization of passive voice and we illustrate the approach by analysing the passive voice contribution to the expression of the function of participation in two typologically and genetically related languages, French and Spanish.
\end{abstract}

La grammaire traditionnelle concevait la voix comme une catégorie verbale au même titre que le temps, le mode, la personne ou le nombre, et

1. Cette étude a été réalisée dans le cadre du Grup de recerca singular, Comunicació $i$ oralitat. Anàlisi experimental de l'oralitat en el contacte de llengües $i$ en els trastorns del llenguatge (2009SGR-1201) et de différents projets de recherche (I+D) financés par le Ministerio de Ciencia y Tecnología (ref. HUM2004-03764; HUM2007-61648; FFI2010-17917).

* Roser Gauchola est professeure à l'Université autonome de Barcelone (Espagne). 
la voix passive comme la simple transformation d'une phrase active de base. Cette conception de la voix passive, comme au demeurant d'une manière générale la plupart des approches plus ou moins formalistes des langues, que ce soit dans le cadre de la grammaire générativetransformationnelle, de la grammaire applicative (Desclés \& Guentchéva, 1993), voire du lexique-grammaire (Leclère, 1993) ou de la théorie des opérations énonciatives (Culioli, 1971), a permis de décrire, souvent de façon exhaustive, certains phénomènes de surface à l'intérieur d'un système donné. Il en est ainsi de la relation entre les énoncés passifs et leurs corrélats actifs, des contraintes de passivation de certains verbes prototypiquement transitifs, ainsi que des rapports entre voix passive et aspect, entre autres. Certaines de ces approches ont également permis de dresser des typologies des langues à partir de l'analyse de certaines structures linguistiques de surface.

Toutefois, si les études formalistes présentent sans aucun doute un intérêt certain, dans une perspective contrastive, elles s'avèrent limitées et peu opérationnelles, voire irréalisables, en particulier lorsque la structure analysée n'existe pas telle quelle dans une des langues prises en compte.

La typologie linguistique fonctionnelle adopte un point de vue radicalement différent qui permet de conjuguer deux domaines d'analyse apparemment incompatibles mais qui s'avèrent de fait complémentaires : la recherche des universaux du langage (i.e. l'analyse de l'invariance) et la recherche typologique des langues (l'étude de la variabilité intralinguistique et interlinguistique). Elle se situe dans une perspective téléonomique des langues, dans la mesure où chaque langue constitue un système de résolution de problèmes cognitifs et communicatifs qui sont universels : les «fonctions» dans la terminologie de la linguistique typologique fonctionnelle. Face à ces fonctions qui leur sont communes, chaque langue apporte ses solutions particulières, ses «techniques", qui sont constituées par les structures propres à cette langue et qui entretiennent des relations d'équilibre à l'intérieur de chaque système. L'approche typologique fonctionnelle s'avère ainsi opérationnelle dans l'analyse translinguistique : elle permet non seulement de répertorier les différentes «techniques» apportées par chaque langue mais encore et surtout de les contraster en expliquant la fonction qu'elles réalisent ${ }^{2}$.

2. Il va sans dire que, si toutes les langues du monde ne possèdent pas toutes les techniques décelées (qui sont particulières à chaque type de langue analysé), 
En adoptant ce point de vue, la voix passive a pu être envisagée comme une sous-technique de la technique «orientation» dont disposent certaines langues pour résoudre un problème cognitif et communicatif universel, la fonction de «participation» (Seiler, 1988), i.e. le rapport entre le noyau d'un évènement (le verbe, le «participé») et les satellites de ce noyau (les syntagmes nominaux, les «participants»).

Dans la technique "orientation», i.e. "the marking of relations of the participants to the participe in the most general sense» (Seiler, 1988 : 13), la voix constitue le moyen grammaticalisé le plus fréquent ${ }^{3}$ de la visée communicative qui peut se traduire par une mise à l'arrièreplan (backgrounding, selon les termes de Hansjakob Seiler) ou par la mise au premier plan (foregrounding). La technique «orientation» peut inclure les voix active, moyenne, médio-passive, réfléchie, réciproque et passive, dont les frontières sont loin d'être nettes, notamment dans les langues indo-européennes.

Il y a lieu dès lors de préciser, dans le cadre de la technique «orientation», en quoi consiste la spécificité de la voix passive dans sa contribution à l'expression de la fonction de "participation», ce qui doit permettre de caractériser la voix passive d'un point de vue fonctionnel et de transcender les caractéristiques formelles stricto sensu. Pour illustrer la démarche, nous analyserons cette contribution de la voix passive à l'expression de la fonction de «participation» dans deux langues typologiquement proches et génétiquement apparentées, le français et l'espagnol.

\section{Caractérisation fonctionnelle de la voix passive}

La technique «orientation» est conçue comme la manipulation des participants selon leur importance relative dans la relation de participation

il n'en reste pas moins que les techniques «absentes» dans une langue peuvent ne pas être pertinentes pour cette langue, être «compensées» par des techniques «voisines» ou tout simplement avoir une manifestation opaque, que la comparaison interlinguistique, en se situant au plan des fonctions, peut aider à mettre en lumière.

3. Dans cette perspective, à l'instar de l'école typologique de Léningrad, on distinguera deux termes, souvent pris comme synonymes dans les recherches syntaxiques, d'une part la diathèse (configuration argumentale qui est propre à chaque verbe, voire à chaque emploi d'un verbe) et d'autre part, la voix, i.e. le moyen le plus grammaticalisé de la diathèse. 
(Seiler, 1988 : 54). Il y a lieu de définir dès lors en quoi consiste cette manipulation dans la voix passive.

Dans la plupart des recherches, la voix passive est considérée comme un cas marqué de la voix active non seulement parce que dans bien des langues le verbe dit «passif» subit des variations morphologiques (processus d'auxiliarisation, émergence de morphèmes - dans le cas des conjugaisons synthétiques, entre autres), mais aussi parce que la passivation entraine des modifications du schéma actanciel de la voix active. Ainsi, l'action prototypique, généralement associée à la voix active, «est celle qui est exercée volontairement par un agent humain qui en garde la commande («control»), action menée jusqu'à son terme et affectant un objet aussi complètement que possible» (Lazard, 1994 : 261).

Partant de cet axiome, les recherches typologiques ont, par ailleurs, permis de montrer qu'il existe une hiérarchie, une structure scalaire entre deux pôles des éléments linguistiques établie selon qu'il est plus ou moins probable qu'ils coïncident avec le sujet/agent (zone gauche de l'échelle ci-dessous) ou avec l'objet/patient (zone droite de l'échelle) (Seiler, $1988: 53$ ) :

$1^{\mathrm{re}}$ personne $>2^{\mathrm{e}}$ personne $>3^{\mathrm{e}}$ personne $>$ Nom propre $>$ humain $>$ animé $>$ inanimé $\mathrm{SUJ} / \mathrm{AG}$

$\mathrm{OB} / \mathrm{PAT}$

Toujours selon Gilbert Lazard (ibid.), «dans la phrase biactancielle prototypique l'agent est thématique, le verbe et l'objet rhématiques».

L'échelle ci-dessus peut dès lors être extrapolée à un continuum entre les deux pôles communicatifs thème/rhème :

$1^{\text {re }}$ personne $>2^{\mathrm{e}}$ personne $>3^{\mathrm{e}}$ personne $>$ Nom propre $>$ humain $>$ animé $>$ inanimé THÈME

RHÈME

L'approche typologique fonctionnelle permet ainsi de caractériser l'action prototypique par un faisceau de traits qui relèvent de la syntaxe (type de fonction syntaxique), de la sémantique (type d'argument) et de la visée communicative (degré de saillance communicative).

Dans la «manipulation» de la relation de participation propre à la voix passive, c'est le patient (voire, dans certains cas, le bénéficiaire ou la cible) qui devient non seulement le sujet du verbe mais aussi et surtout le participant privilégié du point de vue communicatif dès lors qu'il occupe le premier plan et devient de ce fait le thème de l'énoncé, ce qui montre bien que la passivation est un phénomène «multifactoriel» (Givón, 1981) : 
[...] when the agent is pragmatically downgraded and the patientor some other non-agent argument-is thematically more important, as in the prototype passive, agent-related transitive properties are not focused on. Rather, the patient-related, stative-resultative aspects of the events are now in focus, i.e. more salient. And the unimportant agent is more likely to be non-referring and unindividuated. (Givón, 1990 : 567)

Ce phénomène de thématisation du patient caractéristique de la voix passive est à relier à un des facteurs cognitifs et psychologiques intrinsèques à la communication humaine relevés par Scott DeLancey (1980), le «flux attentionnel», qui détermine la disposition des arguments dans l'énoncé, ordonnés en fonction de l'intention communicative du locuteur, qui prétend que son auditeur suive l'énoncé d'une certaine façon. C'est ce qui explique que certaines phrases soient naturelles comme (1), alors que d'autres, quoique grammaticalement correctes, s'avèrent peu usitées ; c'est le cas de l'exemple (2) :

(1) Je vais de Londres à Paris.

(2) Je vais à Paris depuis Londres.

La passivation constitue un exemple de manipulation du flux attentionnel dans la mesure où l'ordre naturel des arguments de l'énoncé $(\mathrm{AG}+\mathrm{V}+\mathrm{PAT} / \mathrm{BEN})$ est inversé pour privilégier un élément, le patient (ou le bénéficiaire, voire la cible) qui apparait avant l'argument censé être communicativement plus «saillant», l'agent. L'analyse typologique fonctionnelle a d'ailleurs permis de montrer que, dans les langues qui possèdent des structures passives, non seulement l'agent est souvent relégué à l'arrière-plan, mais qu'il est le plus souvent effacé ${ }^{4}$. Ainsi donc, si la voix passive partage bien avec d'autres structures - comme la dislocation à gauche - la thématisation d'un participant différent de l'agent, ce qui distingue la voix passive de ces autres constructions, c'est l'effacement de l'agentivité (soit en omettant l'agent, soit en le reléguant à l'arrière-plan communicatif). Les constructions passives rejoignent sur ce point les structures impersonnelles, qui par définition «omettent» l'agent.

4. D'où un des universaux implicatifs concernant la voix passive : si une langue dispose de structures passives avec agent, cette langue a obligatoirement des constructions passives sans agent, l'inverse n'étant pas toujours vrai. 
En adoptant ce point de vue, nous procèderons ci-après à l'analyse comparative des différentes techniques passivantes du français et de l'espagnol.

\section{La technique de la passivation en français}

La langue française dispose de deux grands types de structures grammaticales pour exprimer la passivation : des constructions périphrastiques, formées à l'aide d'auxiliaires plus ou moins grammaticalisés, et les constructions dites «réflexives».

\subsection{Les constructions périphrastiques}

Pour ce qui est du premier type, la construction passive qui a été la plus analysée (la seule d'ailleurs prise en compte par la grammaire traditionnelle) est \{auxiliaire être + participe passé d'un verbe transitif direct\}, considérée comme la «construction de base du passif» (Desclés \& Guentchéva, 1993 : 76). Cette construction admet tout aussi bien la mention de l'agent (i.e. du cas oblique) que l'omission de cet argument :

(3) Le voleur a été arrêté (par la police).

D'autre part, l'élément thématisé, toujours le patient de l'action dans la relation de prédication, peut être assumé par un argument animé, mais aussi par un substantif inanimé :

(4) La banque a été cambriolée.

Le français admet la transformation passive de la plupart des verbes transitifs directs, mais les possibilités formelles offertes par le système fournissent des résultats qui, tout en étant grammaticalement corrects, présentent de fortes contraintes d'utilisation. Ainsi, (5) s'avère beaucoup plus naturel que son corrélat passif (6) :

(5) J'ai lavé la voiture.

(6) La voiture a été lavée par moi ${ }^{5}$.

5. De fait, la phrase (5) pourrait peut-être constituer un énoncé «communicativement vraisemblable» dans une situation de communication où, dans un acte de parole 1, l'opération «laver la voiture» aurait été attribuée à quelqu'un d'autre; autrement dit, l'énoncé (5) viendrait réfuter l'affirmation 
«L'anomalie» représentée par la voix passive ne semble donc pas être due à un «manque» du système mais au fait que cet énoncé enfreint la hiérarchie de thématisation présentée dans la section 1 en élevant au premier rang communicatif une entité non animée (la voiture) au détriment d'un être animé. L'actualisation du système (i.e. l'éventail de solutions syntaxiques) est subordonnée à la fonction que ces structures sont censées exprimer, et lorsqu'il existe un conflit entre le plan syntaxique et le plan communicatif, c'est le facteur pragmatique et communicatif qui l'emporte.

Outre la construction passive de base, la langue française dispose de quatre autres constructions périphrastiques : $\{$ se faire + infinitif $\}$, \{se laisser + infinitif $\}$, $\{$ se voir + infinitif $\}$ et $\{$ s'entendre + infinitif $\}$. La construction $\{$ se faire + infinitif $\}$ admet aussi bien la présence que l'absence de l'agent :

(7) Je me suis fait attaquer dans le métro (par une bande de voyous).

Ce tour présente, toutefois, deux spécificités par rapport à la construction passive dite non marquée. D'une part, il s'agit d'une tournure extrêmement productive à l'oral qui est généralement réservée à un patient humain ${ }^{6}$ et dans laquelle on peut distinguer différents degrés de «responsabilité» du participant humain (Le Goffic, 1993) présentés par ordre décroissant dans les exemples ci-dessous :

(8) Daniel s'est fait psychanalyser.

(9) Daniel s'est fait engueuler par son chef pour ses retards réitérés.

(10) L'escroc s'est fait attraper par la police.

(11) Daniel s'est fait licencier malgré son dévouement pour l'entreprise.

D'autre part, \{se faire + infinitif\} permet de thématiser le bénéficiaire de l'action (qui en est en même temps le causateur) lorsque celle-ci est le résultat d'un acte de volition :

précédemment émise par l'interlocuteur. En tout état de cause, la «solution linguistique» de la thématisation de l'agent au moyen du clivage («c'est moi qui ai lavé la voiture») semblerait, même dans ce cas-là, plus «idoine» du point de vue communicatif.

6. Quoique le discours journalistique, qui est souvent à la base de néologismes ou de nouveaux emplois de structures déjà existantes, fournisse des exemples où l'élément thématisé correspond à un inanimé (cf. La neige se fait attendre dans les Alpes, Le Monde). 
(12) Daniel s'est fait construire une maison à la campagne.

(13) Daniel s'est fait réparer sa moto.

En revanche, lorsque l'action est le résultat d'un processus indépendant de la volonté (et du contrôle) du participant, l'élément thématisé correspond à la cible de l'action :

(14) Je me suis fait voler ma bicyclette.

Le français dispose également d'une troisième technique périphrastique de passivation : la structure \{se laisser + infinitif\}. La signification du lexème laisser explique le glissement sémantique vers la passivation. Dans cette périphrase, le sémantisme («ne pas empêcher, permettre») du noyau lexical de départ est toujours présent, mais il y a lieu de distinguer différents stades dans le processus de grammaticalisation.

L'élément thématisé (la plupart du temps le patient du schéma actanciel) peut renvoyer à un animé, et l'action «subie» n'est pas le résultat d'un acte volontaire du participant privilégié :

(15) Daniel s'est laissé attendrir/impressionner/persuader.

(16) Ne vous laissez pas faire, réclamez, ripostez (Petit Robert Électronique, désormais PRE).

Il en est de même lorsque le participant mis au premier plan correspond à la cible de l'action :

(17) Je me suis laissé dire que... (PRE).

Cependant, dans certains emplois, le patient thématisé est inanimé, ce qui indique que le sémantisme du verbe laisser renvoyant à la permission s'est estompé ou a carrément disparu et que le processus d'auxiliarisation et de grammaticalisation a atteint un stade plus avancé : le lexème s'est complètement désémantisé et il a incorporé une nuance relevant de la modalité aléthique :

(18) Ce vin se laisse boire (PRE).

(19) Ce film se laisse voir (PRE).

Finalement, deux autres constructions périphrastiques avec des verbes du domaine sensoriel ont émergé en français : \{se voir / s'entendre + infinitif\}, où l'élément thématisé renvoie à un animé, voire à un humain. Dans le cas de $\{$ se voir + infinitif $\}$, l'argument occupant le premier plan communicatif peut correspondre aussi bien au patient (ex. 23) qu'à la cible (24) : 
(20) À sa grande stupeur, le romancier s'est vu citer en justice (Duhamel) (PRE).

(21) Elle s'est vu refuser l'entrée au club (PRE).

En revanche, le verbe s'entendre, qui implique un verbe de locution, semble privilégier la thématisation de la cible :

(22) Hier je me suis entendue [sic] dire par le psy de mon fils que lui et moi avions un gros problème (<www.forum.aufeminin.com $>$ ).

Quoiqu'il s'agisse de tournures moins grammaticalisées que celles qui ont été précédemment analysées, le «choix» des lexèmes susceptibles de devenir auxiliaires passifs ne semble pas avoir été arbitraire puisque voir et entendre renvoient à des évènements plutôt qu'à des activités sensorielles par opposition à regarder et écouter qui, eux, impliquent une activité et donc un agent (un actant animé qui est à la recherche d'un stimulus visuel ou auditif).

\subsection{La construction réflexive}

Outre les constructions périphrastiques analysées, la langue française présente un deuxième type de «technique» passive, la construction réflexive ${ }^{7}$ :

(23) Ce vin se boit au dessert (PRE).

Afin de délimiter les usages de cette construction comme tournure passive dont les frontières avec les catégories voisines (cf. note 8) s'avèrent assez floues, nous avons entrepris une recherche, en cours de réalisation, qui a consisté à dépouiller partiellement le PRE en relevant quelque 70 verbes qui sont susceptibles de devenir passifs dans une construction réflexive. Bien que dans l'état actuel de la recherche nous ne puissions émettre que des hypothèses provisoires, il semblerait que pour que l'interprétation passive puisse s'imposer, non seulement le verbe devrait être transitif à la voix active mais encore l'agent devrait renvoyer à un humain et le patient à un inanimé. Toujours est-il que, comme dans les structures analysées plus haut, le tour réflexif permet

7. Celle-ci se caractérise par son caractère polyfonctionnel puisqu'elle peut constituer, comme cela a été souligné par différents auteurs (Desclés \& Guentchéva, op . cit.; Creissels, 2006), un schéma de participation réfléchi, réciproque, moyen, médio-passif ou passif. 
de privilégier le patient et ce d'autant mieux que la forme réflexive omet l'agent du schéma de participation à la voix passive, soit parce qu'il renvoie à un quantifieur universel («Tout le monde boit ce vin au dessert»), soit parce qu'il est difficilement identifiable («On boit ce vin au dessert») ou inconnu. Cet effacement progressif de l'agentivité permet de relier ce type de constructions aux constructions impersonnelles.

\section{Les techniques de la passivation en espagnol}

En espagnol, on décèle, comme en français, deux types de structures passivantes : les tours périphrastiques et la construction réflexive.

\subsection{Les constructions périphrastiques}

L'espagnol dispose de la construction analytique, directement héritée du latin, formée à l'aide de l'auxiliaire $\{$ ser + participe passé\}, qui admet aussi un cas oblique :

(24) El duque ha sido acusado de desviación de fondos (por el fiscal). [Glose : Le duc a été accusé de détournement de fonds (par le procureur).]

L'espagnol possède également une technique périphrastique proche de $\{$ se laisser + infinitif\}, la structure $\{$ dejarse + infinitif $\}$, où le verbe conjugué contient dans son sémantisme l'idée d'absence d'opposition. La plupart du temps, l'élément thématisé, qui correspond au patient, est animé (ex. 25 et 26), mais la possibilité d'avoir un patient inanimé (le tour ayant, comme en français, une nuance de modalité aléthique) n'est pas à exclure (cf. 27) :

(25) Daniel se ha dejado engañar. [Daniel s'est laissé tromper.] ${ }^{8}$

(26) Daniel se deja querer. [Daniel se laisse désirer.]

(27) Esa película se deja ver. [Ce film se laisse voir.]

Néanmoins, malgré cette similarité formelle entre les deux langues résultant, en particulier pour la première structure, de leur origine commune, le français et l'espagnol présentent des différences notoires quant à l'usage que ces langues font de ce type de structures. En premier lieu,

8. Les énoncés entre crochets correspondent à des gloses des énoncés espagnols et non pas à des traductions en français. 
la langue espagnole comprend un nombre plus limité de "solutions» périphrastiques de passivation que le français. De fait, l'espagnol n'a développé, en ce qui concerne la construction \{se faire + infinitif\}, que la variante où l'élément thématisé coïncide avec le bénéficiaire, qui est aussi le causateur ou déclencheur de l'action :

(28) Daniel se ha hecho construir una casa en el campo. [Daniel s'est fait construire une maison à la campagne.]

En revanche, lorsque l'action déclenchée n'est pas le résultat d'une volition, cette structure semble faire défaut, ce qui est d'ailleurs aussi le cas des tournures avec se voir et s'entendre, si bien que dans le processus de passivation en espagnol, les seuls participants qui ont tendance à être privilégiés du point de vue communicatif sont le patient et le bénéficiaire, ce qui est de fait confirmé par la tournure \{dejarse + infinitif\}, qui admet difficilement, du moins de façon naturelle, la transposition littérale du français 'Il s'est laissé dire que'...

En deuxième lieu, la fréquence d'emploi, notamment à l'oral, des constructions périphrastiques est bien moindre en espagnol qu'en français. Si l'on prend en compte le principe postulé par la typologie fonctionnelle selon lequel tout peut être dit dans toutes les langues du monde, il y a lieu de se demander comment la langue espagnole résout le problème que ce type de «techniques» exprime en français.

Comme cela a été dit plus haut, la fonction remplie par la passivation est double : l'effacement de l'agentivité (le cas oblique pouvant toujours être omis) et la thématisation d'un participant qui, à la voix active, serait relégué à l'arrière-plan communicatif. Or, il s'avère que l'espagnol ne requiert pas une construction passive pour pouvoir thématiser un actant autre que l'agent, comme le montre l'exemple (29), où l'on peut conserver la voix active tout en privilégiant le patient et en reléguant l'agent à l'arrière-plan communicatif :

(29) A mi perro lo ha atropellado un coche [A mon chien l'a renversé une voiture] qui serait l'équivalent le plus naturel dans une situation de communication ordinaire de :

(30) Mon chien a été renversé par une voiture.

Ce déplacement est possible en espagnol car, même si cette langue, tout comme le français, est une langue SVO selon la typologie de Joseph Greenberg (1966), le degré de rigidité syntaxique en espagnol est moindre qu'en français, ce qui est à relier à deux phénomènes propres à la langue espagnole. 
En premier lieu, cette langue a conservé, comme en latin, une grande variabilité flexionnelle verbale : il existe presque pour tous les temps une relation biunivoque entre le suffixe verbal, d'une part, et le temps et la personne, d'autre part. Cette information fournie par la morphologie verbale permet une plus grande souplesse syntaxique ${ }^{9}$. En deuxième lieu, quoique l'espagnol, de même que le français, ait perdu au cours de son évolution la flexion casuelle dans l'assise nominale (substantifs et adjectifs), cette langue n'est pas pour autant dépourvue de marques de cas pour les compléments (qu'ils soient directs animés ou indirects). Dans l'exemple (30) cette marque est double : la préposition $a$ (qui interdit la lecture agentive de cet actant) et la reprise anaphorique par le pronom accusatif $l o^{10}$.

On pourrait arguer que la construction espagnole ne serait qu'une transposition de la dislocation à gauche du français ${ }^{11}$, «technique» utilisée également pour thématiser un argument comme dans l'exemple suivant :

(31) Daniel, je l'ai rencontré ce matin.

Or les deux constructions, quoique servant le même propos, ne sont pas tout à fait équivalentes: d'une part, dans la dislocation à gauche, il y a une rupture syntaxique entre l'élément détaché et l'énoncé qui suit et, d'autre part, cette rupture syntaxique se traduit également au plan suprasegmental par une pause après l'élément thématisé ${ }^{12}$ et, par conséquent, par un accent.

9. Il a été démontré en typologie fonctionnelle que, plus la morphologie est porteuse d'information, plus la syntaxe est libre, alors que la syntaxe a tendance à devenir plus fixe lorsque la morphologie fait défaut à cet égard.

10. La comparaison interlinguistique s'avère encore une fois révélatrice à ce sujet : la langue anglaise, qui manque d'oppositions de genre et de nombre pour l'adjectif, de genre pour le substantif et qui ne présente qu'une opposition résiduelle de personne dans le verbe, n'admet pas la postposition du sujet dans les phrases déclaratives. Le cas extrêmement opposé est celui de l'espagnol, où la syntaxe est beaucoup plus flottante. Entre ces deux pôles se situe le français, morphologiquement plus riche que l'anglais mais beaucoup moins que l'espagnol.

11. Malgré les liens fonctionnels qu'elle entretient avec la voix passive, dans le cadre restreint de cette contribution, nous n'aborderons pas l'analyse de la dislocation à gauche.

12. Cf. «La phrase sera délimitée [...] comme construite sans rupture prosodique» (Muller, $2008: 36$ ). 
En revanche, en espagnol :

(32) A Daniel lo he visto esta mañana. [A Daniel je l'ai vu ce matin.]

Ici il n'y a pas de coupure syntaxique entre l'élément déplacé ( $A$ Daniel) et le reste de l'énoncé. Au plan suprasegmental, il n'y a pas de pause après Daniel et, quoique ce constituant reçoive un accent de mot, la mise en relief de l'élément déplacé est bien moindre qu'en français.

Qui plus est, si en français la coexistence dans un même énoncé de deux éléments (un lexème et un clitique) coréférentiels s'avère impossible (d'où la pause après l'élément disloqué), en espagnol l'effacement du pronom de reprise conduit à une lecture radicalement différente :

(33) A Daniel he visto (y no a Pedro) esta mañana. [A Daniel je l'ai vu (et non pas à Pierre) ce matin.]

Le tour est ainsi interprété comme une topicalisation (emphatique) du rhème (Daniel) et non comme une mise au premier plan du patient (une thématisation), ce qui se traduit également au plan suprasegmental. Il s'ensuit que la thématisation par déplacement du participant en espagnol, comme Hansjacob Seiler (1988 : 12) l'a souligné à propos de la voix passive, «[...] in contrast to topicalization and left-dislocation, is an integral part of the grammar».

Ainsi, la divergence essentielle entre le français et l'espagnol pour ce qui est de la thématisation d'un participant autre que l'agent est due au fait que le français (l'anglais et les langues accusatives ${ }^{13}$ ayant un comportement morphologique où prédomine la neutralisation d'oppositions) a développé et créé des constructions passives pour pouvoir privilégier communicativement ledit participant, alors que l'espagnol, dont la syntaxe est plus libre, peut s'en tenir au déplacement en début d'énoncé de l'argument.

On retrouve ces différences lorsqu'on analyse le comportement de ces deux langues dans des situations où la distinction thème-rhème s'avère particulièrement saillante (Moreno Cabrera, 1991 : 704). Soit les énoncés (34) et (35) et leurs traductions en français (ex. 36 et 37) :

(34) - ¿Qué ha hecho Daniel cuando ha sabido la noticia? - Daniel se ha ido. [- Qu'a fait Daniel quand il a appris la nouvelle? - Daniel est parti.]

13. Par opposition aux langues ergatives. 
(35) - ¿Quién se ha ido, Daniel o Pablo? - Se ha ido Daniel. [- Qui est parti, Daniel ou Paul? - Est parti Daniel.]

(36) - Qu'est-ce que Daniel a fait lorsqu'il a appris la nouvelle? - Daniel est parti.

(37) - Qui est parti, Daniel ou Paul? - C'est Daniel qui est parti.

En (34), le thème est représenté par Daniel, ce qui explique que dans la réponse à la question, l'énoncé commence par le thème, qui coïncide avec l'agent. En (35), le thème de l'énoncé se situe dans le participé et dans la réponse l'énoncé commence également par le thème même s'il coïncide non pas avec le sujet (situation non marquée) mais avec le verbe. Par ailleurs, les réponses aux questions de (34) et (35) sont en distribution complémentaire puisque l'utilisation de l'une ou l'autre est due non pas à une contrainte syntaxique (quel que soit l'ordre des éléments, le rapport syntaxique ne varie pas), mais au flux attentionnel, ce qui montre qu'en espagnol, lorsqu'il y a un «conflit» entre la structure syntaxique et la structure communicative, la construction formelle s'adapte aux contraintes fonctionnelles. Autrement dit, si «tout système linguistique connait deux zones opposées : celle des contraintes (par exemple, les servitudes positionnelles) et celle de la liberté (relative) dont dispose le locuteur pour organiser son message» (Feuillet, 2006 : 57), en espagnol, lorsqu'il y a divergence entre ces deux facteurs, la priorité n'est pas donnée aux fonctions syntaxiques, mais aux éléments les plus saillants du point de vue communicatif.

En français, par contre, cette «adaptation» de la syntaxe à la communication ne se produit pas lorsque le thème de l'énoncé ne coïncide pas avec l'agent du participé (ex. 37). Quand il y a des discordances entre le plan formel et le plan communicatif, la rigidité syntaxique du français empêche la postposition du rhème-agent et au lieu de thématiser le thème (comme c'est le cas de la langue espagnole), le français opte pour focaliser le rhème à l'aide du clivage, ce qui permet de prévenir l'interlocuteur du fait que, contrairement à ce qui est de règle, l'information inconnue sera mise au premier plan communicatif. Ces différences de comportement des deux langues face à un même problème communicatif expliquent les divers degrés de productivité de la voix passive en français et en espagnol.

De ce qui vient d'être souligné, il ne s'ensuit pas néanmoins que la passivation périphrastique ne soit qu'une possibilité virtuelle du système de la langue espagnole qui n'est jamais actualisée dans la parole au sens 
saussurien du terme. Certes, dans la communication orale ordinaire, ce type de constructions n'est guère privilégié, car les mécanismes syntaxiques supposant un coût psycho-cognitif plus important, en raison de leur caractère marqué, tendent à être peu utilisés dans la langue orale ${ }^{14}$ (ce qui est d'ailleurs corroboré par le développement psychogénétique de l'enfant). Cependant, certains discours «spécialisés» ont recours à ces tournures. Ainsi, la construction passive est fréquente en espagnol au début d'un article de presse rapportant un fait divers :

(39) Una mujer fue asesinada ayer por su marido. [Une femme a été assassinée hier par son mari.]

Cet énoncé ne semble pas répondre à la nécessité de thématiser un argument (puisque les deux participants, actualisés par l'article indéfini, sont rhématiques), mais correspond à une formule d'introduction de thème-zéro, qui se rapproche des constructions impersonnelles (cf. Le Goffic, 1993). Dans la même situation de communication, cet énoncé peut être concurrencé par la structure à renversement actanciel, ce qui semble indiquer que le choix de la phrase utilisée dépend du point de vue adopté par le locuteur :

(40) Un hombre asesinó ayer a su mujer. [Un homme a assassiné hier à sa femme.]

$\mathrm{Si}$, comme on l'a vu, d'une part, la langue espagnole présente une tendance moins marquée à l'utilisation de la voix passive et que, d'autre part, elle ne dispose pas de certains tours périphrastiques qui existent en français, il y a lieu de s'interroger sur les procédures adoptées par l'espagnol pour combler ces lacunes. Soient les énoncés :

(41) Je me suis fait attaquer dans le métro.

(42) Je me suis fait voler ma bicyclette.

(43) Daniel s'est fait licencier malgré son dévouement pour l'entreprise.

14. Nous en avons une preuve particulièrement éclairante dans l'interrogation en français : lorsque les trois mécanismes linguistiques mis en œuvre en français pour poser une question totale - l'intonation montante, le morphème interrogatif est-ce que ou l'inversion du sujet clitique - sont possibles, cette dernière «solution»-qui s'avère la plus complexe du point psycho-cognitif puisqu'elle renverse l'ordre canonique des constituants en français - est la moins naturelle dans la langue parlée. 
(44) Daniel s'est fait engueuler par son chef.

(45) Daniel s'est fait engueuler par son chef pour ses retards réitérés.

(46) L'escroc s'est fait attraper par la police.

Les exemples ci-dessus présentent deux cas de figure : des énoncés avec se faire où l'agent est implicite, comme en (40), (41) et (42); et d'autres où ce participant est mentionné, comme en (43), (44) et (45). Pour le premier type d'occurrences, il semble que la solution la plus naturelle en espagnol consiste à conserver la voix active en thématisant le participant privilégié et à conjuguer le verbe à la $3^{\mathrm{e}}$ personne du pluriel, mécanisme très souvent employé dans cette langue pour effacer l'agentivité :

(47) Me han atacado en el metro (trad. de 40). [M'ont attaqué dans le métro.]

(48) Me han robado la bicicleta (trad. de 41). [M'ont volé la bicyclette.]

(49) A Daniel lo han despedido a pesar de su dedicación a la empresa (trad. de 42). [A Daniel l'ont licencié malgré son dévouement à l'entreprise.]

Pour le deuxième type d'énoncés, l'espagnol conserverait la même structure active tout en remplaçant la $3^{\mathrm{e}}$ personne du pluriel par l'agent du schéma de participation ajouté en fin de phrase :

(50) Al ladrón lo cogió la policía (trad. de 45). [Au voleur l'a attrapé la police.]

Comme indiqué plus haut (section 3.2), les phrases (43) et (44) présentent différents degrés de responsabilité du patient, dont l'expression en espagnol, beaucoup plus opaque qu'en français, pourrait être rendue par le choix du verbe : caer, qui accentue la «passivité» du patient, lorsque le degré de responsabilité est moindre, ou ganarse, lorsque le patient, qui devient la cible dans les deux traductions, a dans une certaine mesure «déclenché » l'action :

(51) A Daniel le ha caído una bronca de su jefe. [A Daniel lui est tombée une engueulade de son chef.]

(52) Daniel se ha ganado una bronca de su jefe por sus repetidos retrasos. [Daniel s'est gagné une engueulade de son chef pour ses retards réitérés.] 
En tout état de cause, la «solution» linguistique la plus naturelle de l'espagnol pour exprimer ce degré de responsabilité plus ou moins grand du patient dépendra de chaque situation de communication, ce qui est aussi le cas pour les exemples avec \{se voir + infinitif\} où le tour périphrastique pourrait éventuellement être traduit par ver cómo («voir comment») :

(53) FR. A sa grande surprise, le romancier s'est vu citer en justice > ESP. Para su sorpresa, el novelista vio cómo le citaban ante la justicia.

(54) FR. Elle s'est vu refuser l'entrée au club > ESP. Vio cómo le rechazaban la entrada al club.

Alors que le tour avec s'entendre pourrait avoir un équivalent dans tener que oír («avoir à entendre»), qui est teinté d'une certaine nuance déontique :

(55) FR. Hier je me suis entendu dire par le psy de mon fils que lui et moi avions un problème grave $>$ ESP. Ayer tuve que oír al psicólogo de mi hijo decir que él y yo teníamos un problema grave.

\subsection{Les constructions réflexives}

De même que le français, l'espagnol dispose d'une «technique » réflexive de passivation qui permet de thématiser le patient :

(56) Los pisos se venden muy rápido. [Les appartements se vendent très rapidement.]

(57) Esa sopa se sirve caliente. [Cette soupe se sert chaude.]

Il s'agit en général de schémas actanciels où l'élément thématisé est fortement déterminé (à l'aide d'articles définis ou de démonstratifs, par exemple) et antéposé au "participé». Contrairement à ce que l'on a pu observer pour les périphrases, l'ordre des éléments est plus rigide pour ce type de constructions car la postposition des éléments thématisés pourrait être, dans certains cas, source d'ambiguïté : les énoncés admettraient d'autres lectures où il serait question d'un sujet elliptique (ce que l'espagnol permet pourvu que ce sujet soit communicativement récupérable). Ainsi, les phrases ci-dessous avec postposition des participants déplacés pourraient recevoir différentes interprétations :

(58) Se venden los pisos muy rápido. [«Ils ou elles se vendent les appartements très rapidement», datif réciproque.] 
(59) Se sirve la sopa muy caliente. [ «Il ou elle se sert la soupe très chaude», datif réfléchi.]

La grammaire traditionnelle espagnole distingue, en outre, deux autres types de constructions réflexives : les constructions dites «pasivas reflejas» et les «pasivas impersonales».

Dans les premières, le verbe aurait comme sujet un syntagme nominal indéfini postposé qui s'accorderait avec le verbe :

(60) Se venden pisos. [Se vendent des appartements.]

(61) Se alquila una habitación en la casa de al lado. [Se loue une chambre dans la maison d'à côté.]

(62) Se corearon consignas anti-sistema. [Se hurlèrent des consignes antisystème.]

L'interprétation passive se trouve confortée par le fait que, dans cette structure, il n'est pas courant d'expliciter l'agent bien que cette possibilité ne soit pas à exclure :

(63) Se corearon consignas anti-sistema por parte de los manifestantes. [Se hurlèrent des consignes antisystème de la part des manifestants.]

Le fait que le patient ne soit pas individualisé par détermination montre bien qu'il s'agit d'un élément rhématique et explique la postposition de cet actant par rapport au verbe, ce qui montre de nouveau qu'en espagnol c'est le statut communicatif des participants qui détermine la distribution des constituants plutôt que leur fonction syntaxique (en l'occurrence, le sujet).

Ainsi, la fonction communicative dévolue à la «pasiva refleja» ne correspond pas à la thématisation d'un argument (le patient) mais à l'effacement de l'agentivité, qui est aussi toujours présente, à des degrés divers, dans tout processus de passivation, ce qui permet de relier ce type de tournures aux constructions impersonnelles, où l'effacement atteint son point maximal.

Les «pasivas impersonales» présentent un patient humain introduit par la préposition $a$ et postposé à un verbe conjugué à la $3^{\mathrm{e}}$ personne du singulier :

(64) Se detuvo a los ladrones en el acto. [S'est attrapé aux voleurs surle-champ.] 
(65) Se reclutó a niños soldados para la guerra. [S'est recruté à des enfants soldats pour la guerre.]

À la différence des «pasivas reflejas», les «pasivas impersonales» admettent aussi bien des participants clairement individualisés avec des articles définis ou d'autres actualisateurs (ex.63) que des patients non individualisés (ex.64). Dans le premier cas, la thématisation du patient est viable avec déplacement dudit patient et reprise par un pronom anaphorique sans qu'il y ait pour autant une rupture syntaxique ou intonative dans la phrase :

(66) A los ladrones se les detuvo en el acto. [Aux voleurs se les est attrapés sur-le-champ.]

Dans ce cas, la même opération appliquée à des $\mathrm{SN}$ indéfinis relèverait de la topicalisation du rhème. Sur ce point, les «pasivas impersonales» rejoignent les «pasivas reflejas» en privilégiant l'effacement de l'agentivité au détriment de la thématisation.

\section{Conclusions}

L'approche fonctionnelle, en l'occurrence dans l'analyse de la voix passive en français et en espagnol, permet non seulement de décrire les mécanismes passivants mais encore d'en expliquer l'usage et leurs différents degrés d'applicabilité et de pertinence dans les langues analysées. En effet, la prise en compte de la «fonction» linguistique, intrinsèque aux différentes «techniques », et de la «situation de communication » où elles sont effectivement utilisées, permet de caractériser leur usage selon leur degré de naturalité et en fonction des possibilités syntaxiques et morphologiques de chaque langue.

Il a pu ainsi être constaté que la voix passive, qui traduit une nécessité d'adapter la syntaxe à la communication, est plus productive en français qu'en espagnol. L'espagnol est une langue caractérisée par une syntaxe plus souple et plus conforme au flux attentionnel, où ce type de phrase est relégué à des types discursifs très précis.

Les conclusions susceptibles d'être tirées de l'analyse présentée débordent du cadre strict de l'épistémologie linguistique pour pénétrer dans des domaines d'application où la communication exolingue est de mise. Ainsi, pour ce qui est de la traduction, la recherche a permis de montrer que, quoique certaines des constructions analysées soient «communes » aux deux langues étudiées, la simple transposition formelle ne fournit en espagnol des résultats naturels que dans des 
contextes très spécifiques et que, dans sa tâche, le traducteur (et le linguiste) se doit de procéder par abduction, i.e. doit inférer la «fonction» à partir d'une «technique» de la langue source et la transposer dans une «technique» de la langue cible.

Pour ce qui est des applications dans l'enseignement-apprentissage des langues, si la didactique des langues étrangères a bien montré depuis longtemps la nécessité d'enseigner-apprendre une langue en situation, cette nécessité devient, dans le cas de la voix passive, une exigence incontournable afin de contrecarrer, chez le francophone apprenant l'espagnol, la tendance à un usage linguistiquement correct mais «communicativement excessif» de la voix passive et, chez l'hispanophone apprenant le français, l'utilisation «abusive» de structures actives impersonnelles (avec le pronom indéfini on) dont la «fonction» n'est pas la thématisation de participants autres que l'agent mais l'effacement de l'agentivité.

Les productions linguistiques «déviantes» des apprenants selon leur profil linguistique ne peuvent pas être expliquées par une différence syntaxique entre les deux langues en présence, notamment dans le cas de la structure passive de base, qui existe dans les deux systèmes, mais par l'usage différent qu'en font les locuteurs de ces langues en fonction de leurs besoins communicationnels. L'approche fonctionnelle fournit ainsi une assise théorique non seulement pour caractériser les erreurs des apprenants mais aussi pour établir des procédures didactiques qui contraignent l'apprenant à utiliser la «technique» à apprendre dans une situation de communication vraisemblable.

Dans cette perspective, la linguistique n'est plus un carcan pour la didactique des langues - maternelles ou étrangères d'ailleurs - et ne contraint pas les procédures didactiques, mais devient opérationnelle pour l'analyse contrastive qui nolens volens se réalise dans l'apprentissage d'une langue seconde ou étrangère et permet de réaliser une analyse en amont des manifestations formelles de la langue, i.e. au niveau de la structuration psycho-cognitive et transactionnelle. 


\section{RÉFÉRENCES BIBLIOGRAPHIQUES}

CReissels D. (1995) : Éléments de syntaxe générale, Paris, PUF.

CReISSEls D. (2006) : Syntaxe : une introduction typologique 1 et 2. Catégories et constructions, Paris, Hermès.

Culioli A. (1971) : «À propos d'opérations intervenant dans le traitement formel des langues naturelles », Mathématiques et sciences humaines, 31, 7-15.

DeLancey S. (1980): An Interpretation of Split Ergativity and Related Patterns, Bloomington, Indiana University Linguistic Club.

Desclés J.-P. et Guentchéva Z. (1993) : «Le passif dans le système des voix du français», Langages, 109, 73-102.

Feuillet J. (2006) : Introduction à la typologie linguistique, Paris, Honoré Champion.

François J. et Victorri B. (2000) : La Sémantique du lexique verbal, Syntaxe et sémantique 2, Caen, Presses universitaires de Caen.

Givón T. (1981) : «Typology and Functional Domains », Studies in Language, 5 (2), 163-193.

Givón T. (1990) : Syntax. A Functional Typological Introduction. Vol.II, Amsterdam/Philadelphie, John Benjamins.

GreENBERG G. (1966) : Language Universals, Mouton, Indiana University. LAZARD G. (1994) : L'Actance, Paris, PUF.

LeCLeRE C. (1993) : "Classes de constructions directes sans passif», Langages, 109, 7-31.

Le Goffic P. (1993) : Grammaire de la phrase française, Paris, Hachette.

MoRENo CABRERA J.-C. (1991) : Curso universitario de lingüística general. Tomo I: Teoría de la gramática y sintaxis general, Madrid, Síntesis.

Muller C. (2008) : Les Bases de la syntaxe, Bordeaux, Presses universitaires de Bordeaux, $2^{\mathrm{e}}$ éd.

SeILer H. (1988) : The Dimension of Participation, Función, 7, Guadalajara, Centro de Investigación de Lenguas Indígenas.

SEILER H. (2000) : Language Universals Research. A Synthesis, Tübingen, Gunter Narr Verlag.

Sinner C. et van Raemdonck D. (2005) : «"Faire" et "se faire", c'est toujours faire? Le médio-factitif en espagnol et en français", dans C. Sinner et G. Veldre (dir.), Diathesen im Französischen / Les Diathèses en français, Francfort-sur-le-Main, Peter Lang, 155-175. 